\title{
Resistance of Human Spermatozoa to Cryoinjury in Repeated Cycles of Thaw-Refreezing
}

\author{
Sidney Verza Jr., Cinthia M. Feijo, Sandro C. Esteves \\ ANDROFERT, Referral Center for Male Reproduction, Campinas, Sao Paulo, Brazil
}

\begin{abstract}
Objective: To study the resistance of human spermatozoa to cryoinjury in repeated cycles of thaw-refreezing by using the fast liquid nitrogen vapor method.

Materials and Methods: Semen specimens were obtained from sixteen normal and oligozoospermic individuals who required disposal at the sperm bank. Five of them had testicular cancer. Specimens were thawed and an aliquot was removed for analysis. The remaining specimens were refrozen without removing the cryomedia. Repeated freeze-thaw cycles were performed until no motile sperm were observed. Sperm motility, number of motile spermatozoa and viability were determined after thawing. Resistance to cryoinjury was compared between groups and also after each refreezing cycle within groups.

Results: Motile spermatozoa were recovered after five and two refreeze-thawing cycles in normozoospermic and oligozoospermic specimens, respectively. There were no significant differences in the recovery of motile spermatozoa between thaws within each group of normal and oligozoospermic specimens, but percentage motility and total number of motile spermatozoa were significantly lower in the oligozoospermic one. Specimens from men with cancer were exposed to six refreeze-thawing cycles. Although recovery of motile spermatozoa was significantly impaired after each thawing, there were no significant differences in the recovery of motile sperm between thaws in cancer and non-cancer groups.

Conclusions: Human spermatozoa resist repeated cryopreservation using the fast liquid nitrogen vapor method. Normozoospermic specimens withstand refreezing for an average two cycles longer than oligozoospermic ones. Specimens from cancer patients seem to resist repeated cryoinjury similarly to non-cancer counterparts. Resistance to repeated cryoinjury was related to the initial semen quality.
\end{abstract}

Key words: sperm; fertility; cryopreservation; oligozoospermia; cancer

Int Braz J Urol. 2009; 35: 581-91

\section{INTRODUCTION}

Since the 1960's, sperm banking has been successfully used to preserve male fertility (1). Efforts have been made to improve cryopreservation of human spermatozoa and to obtain better gametes after thawing. It is well known that the freeze-thawing process affects the fertile potential of human sperm on several aspects. The freeze and thawing process decrease sperm motility
(2) and sperm penetration into the cervical mucus (3), alter cell membrane fluidity $(4,5)$, decrease acrosome integrity (6) and may induce sperm apoptotic deoxyribonucleic acid (DNA) fragmentation (7). Lower fertilization and pregnancy rates are achieved when frozen-thawed spermatozoa are used for intrauterine insemination (8) and conventional in-vitro fertilization (9). However, it has been shown that similar fertilization and pregnancy rates can be obtained with 
intracytoplasmic sperm injection (ICSI) using both frozen-thawed and fresh motile spermatozoa (10).

Indications for sperm banking have expanded in the recent years, but the group of cancer patients in reproductive age deserves special attention. Chemotherapy and radiation are often gonadotoxic and although fertility restoration occurs in about $50 \%$ of cancer survivors, permanent sterility is common $(11,12)$. Therefore, sperm cryopreservation is recommended before therapy $(13,14)$. The main concern, however, is that cancer patients are often urged to start treatment and in most individuals only very few specimens preserved.

Even using modern assisted reproductive techniques (ART), pregnancy rates per attempt are below the $50 \%$ rate $(10,15,16)$. Then, many couples require multiple ART attempts in order to achieve a live birth. When frozen-thawed sperm are used for ART, one or more vials have to be thawed until an adequate number of viable sperm are obtained. However, ART procedures require very few gametes, especially ICSI, and the remaining ones are left over and ultimately discharged. Refreezing thawed specimens would provide additional opportunities for conception in men who have banked a limited number of sperm specimens, such as cancer patients and those who have a small supply of donor semen left from a previous pregnancy and wish to use it for siblings.

The objective of this study was to assess the resistance of human spermatozoa to cryoinjury in repeated cycles of thaw-refreezing.

\section{MATERIALS AND METHODS}

\section{Specimens}

Frozen semen specimens were obtained from 16 subjects who requested disposal from our sperm bank. We included all eligible patients who requested disposal over a 6-year period. Specimens remained in storage for an average time of 2.3 years (interval: 1-6 years). According to the pre-freeze sperm count at the time of banking, specimens were divided as normozoospermic $\left(\geq 20 \times 10^{6} / \mathrm{mL} ; \mathrm{n}=7\right)$ or oligozoospermic $\left(<20 \times 10^{6} / \mathrm{mL} ; \mathrm{n}=9\right)$. Reasons for sperm banking were: (i) enrollment in our ART egg donation program, in which sperm cryopreservation is mandatory to facilitate patient treatment synchronization (n $=8)$; (ii) vasectomy $(\mathrm{n}=3)$, and cancer $(\mathrm{n}=5)$. All cancer patients had testicular malignancies. Two of them have previously had unilateral orchiectomy at the time of banking and were oligozoospermic, while three banked sperm before surgery and were normozoospermic. We have also divided the specimens according to the existence of cancer $(\mathrm{n}=5)$ or not ( $\mathrm{n}$ $=11$ ), in order to compare the resistance of repeated freezing and thawing between them. The study was approved by our Institutional Review Board and informed consent was obtained from all subjects.

\section{Cryopreservation and Thawing Procedures}

At the time of initial freezing, specimens were collected by masturbation after 2-3 days of ejaculatory abstinence into sterile containers. An aliquot of each liquefied specimen was analyzed according to the WHO guidelines (17). Specimens were cryopreserved by the liquid nitrogen vapor method, using TEST-yolk buffer with glycerol as a freezing media, as previously described (4). In brief, a vial of the freezing agent (TEST-yolk buffer with $12 \%$ glycerol, Irvine Scientific, USA) was thawed by incubation at $37^{\circ} \mathrm{C}$. An aliquot of the medium equal to $25 \%$ of the original specimen volume was then added to the specimen. The specimen was gently mixed for 5 minutes using an aliquot mixer. This process was repeated until the ratio of freezing medium to ejaculate was $1: 1 \mathrm{v} / \mathrm{v}$. The mixture was loaded into $1.0-\mathrm{mL}$ cryovials, which were placed at $-20^{\circ} \mathrm{C}$ for 8 minutes and submerged in liquid nitrogen vapor at $-79^{\circ} \mathrm{C}$ for 2 hours. The vials were then plunged into liquid nitrogen for long-term storage at $-196^{\circ} \mathrm{C}$. For thawing, all cryovials from each subject were removed from liquid nitrogen storage dewars and were thawed at room temperature for 5 minutes. Then, cryovials were transferred to a $37^{\circ} \mathrm{C}$ incubator for 20 minutes (4).

\section{Assessment of Sperm Parameters}

After incubation and homogenization of frozen-thawed specimens of each individual patient, 
an aliquot was removed for analysis of sperm motility and total number of motile sperm. Recovery percentages of motile spermatozoa were calculated after each thaw as the ratio between post-thaw and pre-freeze percentages of motile sperm X100. If only immotile sperm were seen after thawing, testing for sperm viability was performed using eosin exclusion dye (17).

\section{Refreeze Procedure}

After removing an aliquot for analysis, the remaining specimen was refrozen by the same freezing method described above, neither removing the cryoprotectant used in the original freezing cycle nor adding new one. The specimens were then stored in liquid nitrogen for at least 48 hours and thawed using the method previously described. The thaw-refreeze cycles were repeated until no motile sperm were obtained.

\section{Statistical Analysis}

Data are expressed as mean $\pm \mathrm{SD}$. The Student's unpaired t-test was used to analyze statistical differences in pre-freeze sperm parameters and in percentage motility, percentage recovery of motile sperm and total number of motile spermatozoa in normozoospermic and oligozoospermic groups, and also in cancer and non-cancer specimens after each thaw. The Student's paired t-test was used to compare percentage motility, percentage recovery of motile sperm and total number of motile spermatozoa before and after each refreezing cycle within groups. Data were tested for homogeneity and normality by the Kolmogorov-Smirnov test. An alpha level of 0.05 or less was considered significant. Statistical analysis was performed using the Statistica ${ }^{\circledR}$ software package (USA).

\section{RESULTS}

The mean \pm SD age of normozoospermic and oligozoospermic individuals were $36.0 \pm 8.0$ and $29.9 \pm 4.2$ years, respectively $(\mathrm{P}=0.52)$. The length of time samples remained in storage prior to use was not different between groups (Table-1). Pre-freeze sperm parameters are shown in Table-1. Recovery of motile spermatozoa was observed through 5 and 2 refreeze-thawing cycles in normozoospermic and oligozoospermic specimens, respectively (Table-2). A significant decrease in sperm motility and number of total motile spermatozoa was observed after each refreeze-thawing cycle in both normozoospermic and oligozoospermic groups (Table-2, $\mathrm{p}<0.05)$. There

Table 1 - Pre-freeze sperm parameters, length of time samples remained in storage prior to use and number of cancer patients in each group of normozoospermic and oligozoospermic sperm specimens. Values are mean $\pm S D$.

\begin{tabular}{lccc}
\hline & $\begin{array}{c}\text { Normozoospermic } \\
(\mathrm{N}=7)\end{array}$ & $\begin{array}{c}\text { Oligozoospermic } \\
(\mathrm{N}=9)\end{array}$ & p Value* \\
\hline Volume (mL) & $2.6 \pm 0.9$ & $2.5 \pm 1.0$ & 0.83 \\
Sperm count $\left(\mathrm{X} 10^{6} / \mathrm{mL}\right)$ & $103.1 \pm 101.3$ & $4.4 \pm 6.0$ & $<0.01$ \\
Motility (\%) & $64.7 \pm 18.4$ & $48.6 \pm 23.7$ & 0.16 \\
Progressive motility (\%) & $54.8 \pm 19.2$ & $37.0 \pm 23.0$ & 0.12 \\
Leukocytes (X10 $/ \mathrm{mL})$ & $0.2 \pm 0.3$ & $0.1 \pm 02$ & 0.43 \\
Strict morphology (\%) & $11.3 \pm 4.1$ & $8.6 \pm 7.7$ & 0.41 \\
Storage time (years) & $2.3 \pm 0.9$ & $2.4 \pm 1.0$ & 0.79 \\
Cancer patients (N) & $3 / 7$ & $2 / 9$ & -- \\
\hline
\end{tabular}

* comparison between normozoospermic and oligozoospermic groups. 


\section{Repeated Thaw-Refreezing of Human Sperm}

Table 2 - Percentage motility, percentage recovery for motility between thaws and number of total motile sperm after each thaw in both normozoospermic and oligozoospermic groups after repeated thaw-refreezing. Values are mean $\pm S D$.

\begin{tabular}{|c|c|c|c|}
\hline & $\begin{array}{c}\text { Normozoospermic } \\
(\mathrm{N}=7)\end{array}$ & $\begin{array}{l}\text { Oligozoospermic } \\
\qquad(\mathrm{N}=9)\end{array}$ & $\mathbf{p}^{1}$ Value \\
\hline \multicolumn{4}{|l|}{ 1st Thaw } \\
\hline motility (\%) & $50.6 \pm 17.5^{\mathrm{a}}$ & $11.9 \pm 14.1^{\mathrm{g}}$ & $<0.01$ \\
\hline recovery (\%) & $78.0 \pm 10.0^{\mathrm{j}}$ & $20.7 \pm 18.0^{\mathrm{p}}$ & $<0.001$ \\
\hline motile count $\left(\mathrm{X} 10^{6}\right)$ & $88.3 \pm 27.6^{\mathrm{s}}$ & $1.3 \pm 2.0^{\alpha}$ & $<0.001$ \\
\hline \multicolumn{4}{|l|}{ 2nd Thaw } \\
\hline motility (\%) & $26.9 \pm 16.0^{b}$ & $2.4 \pm 4.0^{\mathrm{h}}$ & $<0.01$ \\
\hline recovery $(\%)$ & $55.0 \pm 23.0^{\mathrm{k}}$ & $15.0 \pm 17.8^{q}$ & $<0.001$ \\
\hline motile count $\left(\mathrm{X} 10^{6}\right)$ & $50.1 \pm 31.6^{t}$ & $0.3 \pm 0.6^{\beta}$ & $<0.001$ \\
\hline \multicolumn{4}{|l|}{ 3rd Thaw } \\
\hline motility (\%) & $11.4 \pm 7.8^{\mathrm{c}}$ & $0.4 \pm 0.9^{\mathrm{i}}$ & 0.01 \\
\hline recovery (\%) & $47.0 \pm 28.0^{1}$ & $11.3 \pm 17.5^{\mathrm{r}}$ & $<0.001$ \\
\hline motile count $\left(\mathrm{X} 10^{6}\right)$ & $20.8 \pm 13.8^{\mathrm{w}}$ & $0.1 \pm 0.0^{\gamma}$ & $<0.001$ \\
\hline \multicolumn{4}{|l|}{ 4th Thaw } \\
\hline motility (\%) & $5.0 \pm 6.9^{d}$ & NA & \\
\hline recovery (\%) & $41.0 \pm 34.0^{\mathrm{m}}$ & NA & \\
\hline motile count $\left(\mathrm{X} 10^{6}\right)$ & $8.5 \pm 12.2^{\mathrm{x}}$ & NA & \\
\hline \multicolumn{4}{|l|}{ 5th Thaw } \\
\hline motility (\%) & $3.0 \pm 5.0^{\mathrm{e}}$ & NA & \\
\hline recovery $(\%)$ & $48.0 \pm 47.0^{\mathrm{n}}$ & NA & \\
\hline motile count $\left(\mathrm{X} 10^{6}\right)$ & $5.3 \pm 9.0^{y}$ & NA & \\
\hline \multicolumn{4}{|l|}{ 6th Thaw } \\
\hline motility (\%) & $0.6 \pm 1.1^{\mathrm{f}}$ & NA & \\
\hline recovery (\%) & $12.0 \pm 13.0^{\circ}$ & NA & \\
\hline motile count $\left(\mathrm{X} 10^{6}\right)$ & $0.9 \pm 2.0^{z}$ & NA & \\
\hline
\end{tabular}

$p^{1}=$ comparison between normozoospermic and oligozoospermic specimens, $p^{2}=$ pairwise comparisons within groups from before freezing to after thaw. $p^{2}={ }^{a} X^{b},{ }^{b} X^{c},{ }^{c} X^{d},{ }^{d} X^{e},{ }^{e} X^{f} P<0.03 ;{ }^{j, k, l, m, n, o}$ not significant; ${ }^{s} X^{t},{ }^{t} X^{w},{ }^{w} X^{x},{ }^{x} X^{y} p<0.02 ;{ }^{g} X^{h},{ }^{h} X^{i} p=0.04 ;{ }^{p, q, r}$ not significant; ${ }^{\alpha} X^{\beta} p=0.01 ;{ }^{\beta} X^{\gamma}$ not calculated.

were no significant differences in percentage recovery of motile sperm between thaws within each group of normozoospermic and oligozoospermic specimens (Table-2). However, percentage motility, percentage recovery of motile sperm and total number of motile spermatozoa were significantly lower in the oligozoospermic group when compared to the normozoospermic one (Table-2, $\mathrm{p}<0.001)$. Motile spermatozoa were found in all normozoospermic specimens up to the third refreeze-thawing cycle. Approximately 
Table 3 - Frequency of recovery of motile spermatozoa after each thaw in both normozoospermic and oligozoospermic specimens, and percentage of viable spermatozoa after recovery of no motile sperm.

\begin{tabular}{lcc}
\hline & $\begin{array}{c}\text { Normozoospermic } \\
(\mathrm{N}=7)\end{array}$ & $\begin{array}{c}\text { Oligozoospermic } \\
(\mathrm{N}=9)\end{array}$ \\
\hline Specimens with motile sperm after each thaw & & \\
1st thaw, N (\%) & $7 / 7(100.0 \%)$ & $9 / 9(100.0 \%)$ \\
2nd thaw, N (\%) & $7 / 7(100.0 \%)$ & $5 / 9(55.5 \%)$ \\
3rd thaw, N (\%) & $7 / 7(100.0 \%)$ & $2 / 9(22.2 \%)$ \\
4th thaw, N (\%) & $7 / 7(100.0 \%)$ & $0 / 9(0.0 \%)$ \\
5th thaw, N (\%) & $4 / 7(57.2 \%)$ & $\mathrm{NA}$ \\
6th thaw, N (\%) & $2 / 7(28.6 \%)$ & $\mathrm{NA}$ \\
7th thaw, N (\%) & $0 / 7(0.0 \%)$ & $\mathrm{NA}$ \\
Specimens with viable sperm after recovery of no motile sperm; N (\%) & $5 / 7(71.4)$ & $2 / 9(22.2)$ \\
Percentage of viable spermatozoa, median (max-min interval) & $2.1(0.0-7.0)$ & $0.7(0.0-5.0)$ \\
\hline
\end{tabular}

$50 \%$ and $25 \%$ of all normozoospermic specimens had motile sperm after the 4th and 5th refreeze-thawing, respectively. However, only $55 \%$ of the specimens had motile spermatozoa after the first refreeze-thawing cycle in the oligozoospermic group (Table-3). Viable spermatozoa were still detected in $71.4 \%(5 / 7)$ normozoospermic and $22.2 \%(2 / 9)$ oligozoospermic specimens when only immotile gametes were found after thawing (Table-3).

The mean \pm SD age of cancer and non-cancer patients were $26.4 \pm 6.0$ and $32.7 \pm 8.3$ years, respectively $(\mathrm{p}=0.03)$. Pre-freeze sperm parameters of cancer and non-cancer specimens were not statistically different, except for sperm motility that was higher in the cancer group $(62.5 \pm 18.4$ versus $51.9 \pm$ $21.4, \mathrm{p}=0.04)$. A significant decrease in sperm motility and number of total motile spermatozoa was seen after each refreeze-thawing cycle in both groups, but there were no significant differences in percentage recovery of motile sperm between thaws within each group of cancer and non-cancer specimens (Table-4). Moreover, percentage motility, motility recovery and total number of motile spermatozoa were comparable in cancer and non-cancer groups (Table-4). Recovery of motile spermatozoa was seen through six and five refreeze-thawing cycles in specimens from individuals with and without cancer, respectively (Tables 4 and $5)$.

\section{COMMENTS}

Cryosurvival of human sperm depends mainly on the cryoprotectant, the freezing technique and the initial quality of the specimen. The use of glycerol to prevent injury to human spermatozoa during cryopreservation is well established (18), and its association with buffers, such as Tris (hydroximethyl amino metano) and TES (n-Tris [hydroximethyl] methyl-2-amino-ethane sulphonic acid), and eggyolk yield optimal cryosurvival rates (19). Slow freezing using programmable freezing machines or fast freezing, as used in this study, seems to have no direct effect on thaw survival both in normal and poor quality sperm (20). It has been demonstrated that, independently from the freezing technique, motility from poor quality sperm is kept constant during the first 3 hours after thawing, but it is drastically reduced by the end of an incubation period of 24 hours (20). In this study, we used a rapid vapor freezing method because it is less expensive, time-consuming and labor-intensive, and it has proven equally effective in the recovery of post-thaw motile sperm $(20,21)$.

Studies focusing on the resistance of human spermatozoa to cryoinjury after repeated thaw-refreezing cycles are scarce. Polcz et al., studying only normozoospermic men, first demonstrated the ability of human spermatozoa to resist cryoinjury in succes- 


\section{Repeated Thaw-Refreezing of Human Sperm}

Table 4 - Percentage motility, percentage recovery of motile sperm between thaws and number of total motile sperm after each thaw in both cancer and non-cancer patients after repeated thaw-refreezing. Values are mean $\pm S D$.

\begin{tabular}{|c|c|c|c|}
\hline & $\begin{array}{l}\text { Cancer } \\
(\mathrm{N}=5)\end{array}$ & $\begin{array}{l}\text { Non-cancer } \\
(\mathrm{N}=11)\end{array}$ & $\mathbf{p}^{1}$ Value \\
\hline \multicolumn{4}{|l|}{ 1st Thaw } \\
\hline motility (\%) & $49.0 \pm 21.3^{\mathrm{a}}$ & $29.6 \pm 21.3^{\mathrm{g}}$ & NS \\
\hline recovery $(\%)$ & $72.7 \pm 27.1^{1}$ & $49.3 \pm 41.4^{\mathrm{r}}$ & NS \\
\hline motile count (X106) & $17.9 \pm 13.0^{1}$ & $17.9 \pm 29.4^{7}$ & NS \\
\hline \multicolumn{4}{|l|}{ 2nd Thaw } \\
\hline motility (\%) & $24.2 \pm 22.0^{\mathrm{b}}$ & $11.1 \pm 10.9^{\mathrm{h}}$ & NS \\
\hline recovery $(\%)$ & $45.6 \pm 29.7^{\mathrm{m}}$ & $36.2 \pm 26.5^{\mathrm{s}}$ & NS \\
\hline motile count (X106) & $9.6 \pm 10.5^{2}$ & $9.6 \pm 16.3^{8}$ & NS \\
\hline \multicolumn{4}{|l|}{ 3rd Thaw } \\
\hline motility (\%) & $12.0 \pm 9.9^{c}$ & $5.2 \pm 3.4^{\mathrm{i}}$ & NS \\
\hline recovery $(\%)$ & $51.0 \pm 30.9^{n}$ & $38.9 \pm 22.4^{\mathrm{t}}$ & NS \\
\hline motile count (X106) & $5.0 \pm 4.7^{3}$ & $3.7 \pm 7.0^{9}$ & NS \\
\hline \multicolumn{4}{|l|}{ 4th Thaw } \\
\hline motility (\%) & $5.8 \pm 8.3^{\mathrm{d}}$ & $2.1 \pm 0.8^{\mathrm{j}}$ & NS \\
\hline recovery $(\%)$ & $27.3 \pm 33.0^{\circ}$ & $38.4 \pm 41.4^{\mathrm{u}}$ & NS \\
\hline motile count (X106) & $2.5 \pm 3.6^{4}$ & $0.6 \pm 1.0^{10}$ & NS \\
\hline \multicolumn{4}{|l|}{ 5th Thaw } \\
\hline motility (\%) & $3.6 \pm 6.0^{\mathrm{e}}$ & $1.3 \pm 0.6^{\mathrm{k}}$ & NS \\
\hline recovery $(\%)$ & $45.6 \pm 38.5^{p}$ & $66.7 \pm 57.7^{\mathrm{w}}$ & NS \\
\hline motile count (X106) & $1.6 \pm 1.5^{5}$ & $0.3 \pm 0.7^{11}$ & 0.02 \\
\hline \multicolumn{4}{|l|}{ 6th Thaw } \\
\hline motility (\%) & $0.8 \pm 1.3^{\mathrm{f}}$ & NA & \\
\hline recovery $(\%)$ & $23.2 \pm 22.3^{q}$ & NA & \\
\hline motile count (X106) & $0.3 \pm 0.3^{6}$ & NA & \\
\hline
\end{tabular}

$p^{l}=$ comparison between cancer and non-cancer patients, $p^{2}=$ pairwise comparisons within groups from before freezing to after thaw. $p^{2}={ }^{a} X^{b},{ }^{b} X^{c},{ }^{c} X^{d},{ }^{d} X^{e},{ }^{e} X^{f},{ }^{g} X^{h},{ }^{h} X^{t},{ }^{i} X^{J},{ }^{j} X^{k p}<0.05$; ${ }^{l, m, n, o, p, q, r, s, t, u, w}$ not significant; ${ }^{1,2,3,4,5,6}$ not significant; ${ }^{7,8,9,10,11}$ not significant.

sive thaw-refreeze cycles (22). They observed that spermatozoa were able to withstand five thaw-refreeze cycles and still maintain motility and vitality, although a marked reduction in motility occurred. After the $3 \mathrm{rd}$, 4th and 5th thaw-refreeze cycle, only $3.5 \%, 1.5 \%$ and
$1.8 \%$ motile sperm were seen, respectively. In another study, it has been shown that spermatozoa could resist up to seven cycles thaw-refreeze (23), although a linear decrease of motility per cycle was observed. These authors also compared slow and fast freezing 
Table 5 - Frequency of recovery of motile spermatozoa after each thaw in specimens from patients with and without cancer, and percentage of viable spermatozoa after recovery of no motile sperm.

\begin{tabular}{lcc}
\hline & $\begin{array}{c}\text { Cancer } \\
(\mathrm{N}=5)\end{array}$ & $\begin{array}{c}\text { Non-cancer } \\
(\mathrm{N}=11)\end{array}$ \\
\hline Specimens with motile sperm after each thaw & & \\
1st thaw, N (\%) & $5 / 5(100.0 \%)$ & $11 / 11(100.0 \%)$ \\
2nd thaw, N (\%) & $5 / 5(100.0 \%)$ & $7 / 11(63.6 \%)$ \\
3rd thaw, N (\%) & $5 / 5(100.0 \%)$ & $4 / 11(36.3 \%)$ \\
4th thaw, N (\%) & $3 / 5(60.0 \%)$ & $4 / 11(36.3 \%)$ \\
5th thaw, N (\%) & $2 / 5(40.0 \%)$ & $2 / 11(18.2 \%)$ \\
6th thaw, N (\%) & $2 / 5(40.0 \%)$ & $\mathrm{NA}$ \\
7th thaw, N (\%) & $\mathrm{NA}$ & $\mathrm{NA}$ \\
Specimens with viable sperm after recovery of no motile sperm; N (\%) & $1 / 5(20.0 \%)$ & $6 / 11(54.5 \%)$ \\
Percentage of viable spermatozoa, median (max-min interval) & $0.0(0.0-2.0)$ & $1.0(0.0-7.0)$ \\
\hline
\end{tabular}

techniques, and concluded that fast freezing preserved motility for an average of 2.75 cycles more than slow refreezing. However, their study differs from ours because no information was provided regarding the quality of sperm specimens and slow controlled-freezing was used for the initial freezing cycle instead of the fast liquid nitrogen vapor method. Bandularatne \& Bongso (21) evaluated the extent of sperm cryoinjury up to three repeated freezing in normozoospermic and oligozoospermic men using both slow and fast freezing techniques. They observed a significant reduction in the recovery of motile and viable sperm after each thaw independent of the freezing method. Differences in the recovery of motile and viable sperm between oligozoospermic and normozoospermic specimens were not observed (21).

We also evaluated the resistance to cryoinjury in normozoospermic and oligozoospermic sperm samples, as did Bandularatne \& Bongso (21), but we performed repeated freeze-thawing until absence of post-thaw motile sperm was seen. We observed that recovery of motile spermatozoa was significantly impaired after each refreeze-thawing cycle, but motile sperm was still found after five and two refreezing cycles in normozoospermic and oligozoospermic groups, respectively. Therefore, sperm from normozoospermic men withstood repeated freezing longer than oligoozoospermic ones. Also, there were significant differences in the recovery of motile sperm between thaws when normozoospermic and oligozoospermic groups were compared. We found that the percentage of motile spermatozoa dropped nearly to half after each subsequent freeze-thawing process in normozoospermic specimens, but dropped considerably greater in oligozoospermic ones. A possible explanation for the different results between Bandularatne and Bongso's study and ours may be the fact that they used higher glycerol concentration. In a previous report, we have shown that sperm cryosurvival may be optimized when cryomedia containing $15 \%$ glycerol was used when compared to the standard $12 \%(21)$. In addition, there was a marked difference in the degree of oligozoospermia between studies. Our oligozoospermic group had very low sperm counts (mean count 4.4 million $/ \mathrm{mL}$ ) while theirs had only mild oligozoospermia (mean 15.7 million $/ \mathrm{mL}$ ). Moreover, mean pre-freeze sperm motility was $48.6 \%$ in our group when compared to $73.4 \%$ in theirs. Given that sperm cryosurvival is partially dependent on the semen quality before freezing $(13,25,26)$, we speculate that sperm produced by men with severely defective spermatogenesis is more sensitive to cryoinjury than their normal counterpart is.

Pre-freeze motility of $\geq 15 \%$ can predict a post-thaw motility of $>10 \%$ with $>75 \%$ accuracy (27). Based on these findings, along with the effectiveness of ICSI to achieve successful fertilization with 
very few sperm, cryopreservation is recommended even when a limited number of motile sperm is available (27). In our study, post-thaw recovery of motile sperm that would be adequate for ICSI was obtained even with pre-freeze motility $<12 \%$. Interestingly, all normozoospermic specimens with pre-freeze motility $>10 \%$ had motile sperm after thawing, and pre-freeze motility of 5\% and $3 \%$ yielded recovery of motile sperm after thawing in $50 \%$ and $25 \%$ of the specimens, respectively. Using the same cutoff point of $>10 \%$, only $50 \%$ of oligozoospermic specimens had motile sperm after thawing.

From a clinical standpoint, it would be ideal to compare the results of normal and abnormal samples from men with and without cancer to determine whether cancer patients do indeed fare worse than normozoospermic/oligozoospermic non-cancer patients do. Although our sample size did not allow such comparisons, we have grouped all cancer and non-cancer patients and compared the resistance to repeat freeze-thawing. We found that semen specimens from men with cancer withstood repeated cryoinjury similarly to their counterparts without cancer. Although the recovery of motile spermatozoa was significantly impaired after each refreeze-thawing cycle, there were no significant differences in the recovery of motile sperm between thaws when cancer and non-cancer groups were compared. From our data, it seems that the resistance for repeated cryoinjury is related to the initial semen quality before cryopreservation. However, these results should be interpreted with caution because our cancer population consisted of only five men, and three of them had very good quality semen at the time of banking.

Although it is clear from this study and others that human sperm can resist repeated freezing, the fertilizing ability of such gametes to produce a viable and healthy offspring has not yet been properly addressed. To date, function of refrozen and thawed human spermatozoa has been assessed only by microinjection into zona-intact hamster oocytes (Hamster Intracytoplasmic Sperm Injection test [HICSI test]) (21). Similar fertilization rates were obtained up to the third refreezing cycle when refrozen and fresh spermatozoa were compared $(22.2 \%$ versus $27.3 \%$, respectively) (21). HICSI assay seems to be a reliable indicator for fertilization, but it is not appropriate to predict the occurrence of a viable pregnancy (28). It has been demonstrated that the cryopreservation process induces DNA damage, and that DNA fragmentation is higher in poor quality specimens, such as from oligozoospermic men, when compared to normal ones (7). Oocyte ability to repair double-strand sperm DNA fragmentation is limited, and this type of fragmentation may lead to genomic mutation with consequent embryo alteration. A recent study has shown a significant increase in the percentage of spermatozoa exhibiting DNA fragmentation following the first cycle of refreezing and thawing (29). This study examined the resistance of human sperm up to three refreezing cycles using a slow controlled rate method, and concluded that up to three refreezing cycles can be performed with a level of risk to sperm DNA comparable to that following a single cycle of freezing and thawing, provided that samples are refrozen in their original cryoprotectant and not washed. Therefore, great caution is needed if ICSI is to be performed with thaw-refrozen sperm, and the safety of this procedure has to be extensively studied.

In our study, specimens were cryopreserved with the seminal plasma, and the cryoprotectant used in the first freezing cycle remained throughout the experiment. We observed that refreezing human sperm without processing and/or removing or adding cryoprotectant was fast, simple, and inexpensive. Although we have not examined the effects of removing the seminal plasma before freezing or after thawing on motility, or the role of removing the cryoprotectant and adding a new one after each thawing cycle, Polcz et al. (22) reported marked declines in motility and viability when these washing steps were included and fresh cryoprotectant media was added. Moreover, Thomson et al. (2009) reported that the level of DNA fragmentation more than doubled when the samples were washed and fresh cryoprotectant was added after each thaw, but it only increased slightly when samples were refrozen in the original cryoprotectant without any further treatment (29). In another study by the same authors, a comparable increase in the percentage sperm DNA fragmentation post-cryopreservation was observed both with and without the addition of cryoprotectant (30). Their results seem to indicate that 
the cryoprotectant plays no role in the generation of DNA damage during cryopreservation. On the other hand, the washing steps, which involve dilution and centrifugation, subject the already compromised thawed spermatozoa to many cycles of osmotic shock that may cause mechanical damage to cellular structures and possibly to the DNA molecule $(27,29,30)$. The observed increase in the level of sperm DNA damage and decrease in motility and viability after the freeze-thawing process may be due to a combination of factors, such as the oxidative stress generated via lipid peroxidation, the depletion of protective seminal and spermatozoa antioxidants or the process of cryopreservation itself (27). Removal of seminal fluid via washing steps following the freeze-thawing process may thus deplete protective seminal antioxidants and increase the susceptibility of spermatozoa to oxidative stress.

\section{CONCLUSION}

Human spermatozoa can resist cryoinjury after repeated cycles of cryopreservation using the fast vapor freezing method. Normozoospermic specimens withstand refreezing for an average 2 cycles longer than oligozoospermic ones. Specimens from cancer patients seem to resist repeated cryoinjury similarly to non-cancer counterparts. Sperm ability to resist injury due to the thawing-refreeze process appears to be related to the initial semen quality. Due to the low number of motile sperm and reduced post-thaw motility, gametes that survived repeated freezing would be suitable for intracytoplasmic sperm injection only. Refreezing leftover frozen-thawed specimens may be recommended for patients who were not able to freeze multiple specimens, such as cancer patients and those individuals who have limited supply of donor semen left from a previous pregnancy and wish to use it for siblings. Further studies are required to assess the fertility potential and safety of the use of refrozen human semen for assisted reproduction.

\section{ACKNOWLEDGEMENT}

Mrs. Fabiola Bento edited the manuscript.

\section{CONFLICT OF INTEREST}

None declared.

\section{REFERENCES}

1. Sherman JK: Low temperature research on spermatozoa and eggs. Cryobiology. 1964; 1: 103-29.

2. Centola GM, Raubertas RF, Mattox JH: Cryopreservation of human semen. Comparison of cryopreservatives, sources of variability, and prediction of postthaw survival. J Androl. 1992; 13: 283-8.

3. Fjällbrant B, Ackerman DR: Cervical mucus penetration in vitro by fresh and frozen-preserved human semen specimens. J Reprod Fertil. 1969; 20: 515-7.

4. Esteves SC, Sharma RK, Thomas AJ Jr, Agarwal A: Suitability of the hypo-osmotic swelling test for assessing the viability of cryopreserved sperm. Fertil Steril. 1996; 66: 798-804.

5. Esteves SC, Sharma RK, Thomas AJ Jr, Agarwal A. Evaluation of acrosomal status and sperm viability in fresh and cryopreserved specimens by the use of fluorescent peanut agglutinin lectin in conjunction with hypo-osmotic swelling test. Int Braz J Urol. 2007; 33: 364-74.

6. Cross NL, Hanks SE: Effects of cryopreservation on human sperm acrosomes. Hum Reprod. 1991; 6: 127983.

7. de Paula TS, Bertolla RP, Spaine DM, Cunha MA, Schor N, Cedenho AP: Effect of cryopreservation on sperm apoptotic deoxyribonucleic acid fragmentation in patients with oligozoospermia. Fertil Steril. 2006; 86: 597-600.

8. Peterson EP, Alexander NJ, Moghissi KS: A.I.D. and AIDS--too close for comfort. Fertil Steril. 1988; 49: 209-10.

9. Szczygiel MA, Kusakabe H, Yanagimachi R, Whittingham DG: Intracytoplasmic sperm injection is more efficient than in vitro fertilization for generating mouse embryos from cryopreserved spermatozoa. Biol Reprod. 2002; 67: 1278-84.

10. Kuczynski W, Dhont M, Grygoruk C, Grochowski D, Wolczynski S, Szamatowicz M: The outcome of intracytoplasmic injection of fresh and cryopreserved ejaculated spermatozoa--a prospective randomized study. Hum Reprod. 2001; 16: 2109-13.

11. Lass A, Akagbosu F, Abusheikha N, Hassouneh M, Blayney M, Avery S, et al.: A programme of semen 
cryopreservation for patients with malignant disease in a tertiary infertility centre: lessons from 8 years' experience. Hum Reprod. 1998; 13: 3256-61.

12. Naysmith TE, Blake DA, Harvey VJ, Johnson NP: Do men undergoing sterilizing cancer treatments have a fertile future? Hum Reprod. 1998; 13: 3250-5.

13. Hallak J, Kolettis PN, Sekhon VS, Thomas AJ Jr, Agarwal A: Cryopreservation of sperm from patients with leukemia: is it worth the effort? Cancer. 1999; 85: 1973-8.

14. Agarwal A, Ranganathan P, Kattal N, Pasqualotto F, Hallak J, Khayal S, et al.: Fertility after cancer: a prospective review of assisted reproductive outcome with banked semen specimens. Fertil Steril. 2004; 81: 342-8.

15. Esteves SC, Schneider DT, Verza S Jr. Influence of antisperm antibodies in the semen on intracytoplasmic sperm injection outcome. Int Braz J Urol. 2007; 33: 795-802.

16. Verza Jr S, Esteves SC: Sperm defect severity rather than sperm source is associated with lower fertilization rates after intracytoplasmic sperm injection. Int Braz J Urol. 2008; 34: 49-56.

17. World Health Organization (WHO): Laboratory Manual for the examination of human semen and sperm-cervical mucus interaction. 4th ed., Cambridge, Cambridge University Press. 1999; pp. 68-70.

18. Hammitt DG, Walker DL, Williamson RA: Concentration of glycerol required for optimal survival and in vitro fertilizing capacity of frozen sperm is dependent on cryopreservation medium. Fertil Steril. 1988; 49: 680-7.

19. Hallak J, Sharma RK, Wellstead C, Agarwal A: Cryopreservation of human spermatozoa: comparison of TEST-yolk buffer and glycerol. Int J Fertil Womens Med. 2000; 45: 38-42.

20. Esteves SC, Spaine DM, Cedenho AP, Srougi M: Effects of the technique of cryopreservation and dilution/centrifugation after thawing on the motility and vitality of spermatozoa from oligozoospermic men Int Braz J Urol. 2003; 29: 133-140.

21. Bandularatne E, Bongso A: Evaluation of human sperm function after repeated freezing and thawing. J Androl. 2002; 23: 242-9.

22. Polcz TE, Stronk J, Xiong C, Jones EE, Olive DL, Huszar G: Optimal utilization of cryopreserved human semen for assisted reproduction: recovery and maintenance of sperm motility and viability. J Assist Reprod Genet. 1998; 15: 504-12.

23. Rofeim O, Brown TA, Gilbert BR: Effects of serial thaw-refreeze cycles on human sperm motility and viability. Fertil Steril. 2001; 75: 1242-3.
24. Verza Jr. S, Feijo CM, Schneider DT, Esteves SC: Human cryopreservation: Comparison of three cryoprotectant solutions. Fertil Magazine. 2007; 7: 26-8.

25. Esteves SC, Spaine DM, Cedenho AP: Effects of pentoxifylline treatment before freezing on motility, viability and acrosome status of poor quality human spermatozoa cryopreserved by the liquid nitrogen vapor method. Braz J Med Biol Res. 2007; 40: 985-92.

26. Donnelly ET, McClure N, Lewis SE: Cryopreservation of human semen and prepared sperm: effects on motility parameters and DNA integrity. Fertil Steril. 2001; 76: 892-900.

27. Padron OF, Sharma RK, Thomas AJ Jr, Agarwal A: Effects of cancer on spermatozoa quality after cryopreservation: a 12-year experience. Fertil Steril. 1997; 67: 326-31.

28. Gvakharia MO, Lipshultz LI, Lamb DJ: Human sperm microinjection into hamster oocytes: a new tool for training and evaluation of the technical proficiency of intracytoplasmic sperm injection. Fertil Steril. 2000; 73: 395-401.

29. Thomson LK, Fleming SD, Barone K, Zieschang JA, Clark AM: The effect of repeated freezing and thawing on human sperm DNA fragmentation. Fertil Steril. 2009 [Epub ahead of print].

30. Thomson LK, Fleming SD, Schulke L, Barone K, Zieschang JA, ClarkAM:TheDNAintegrity of cryopreserved spermatozoa separated for use in assisted reproductive technology is unaffected by the type of cryoprotectant used but is related to the DNA integrity of the fresh separated preparation. Fertil Steril. 2009; 92:9911001.

Accepted after revision:

April 6, 2009

\section{Correspondence address:}

Dr. Sandro C. Esteves

Av. Dr. Heitor Penteado, 1464

Campinas, SP, 13075-460, Brazil

Fax: + 5519 3294-6992

E-mail: s.esteves@androfert.com.br 


\section{EDITORIAL COMMENT}

This is an interesting study with the most significant finding being that spermatozoa from cancer patients is capable of withstanding 6 freeze-thaw cycles using the fast liquid nitrogen vapor method. This is important for those cancer patients who are only able to freeze a small amount of semen prior to undergoing treatment, providing more hope for fathering children in their future. The authors also report that the spermatozoa from oligozoospermic men is capable of withstanding significantly fewer freeze-thaw cycles than normozoospermic men and conclude that repeated freezing and thawing is a feasible practice using their method of freezing and that the resistance to cryoinjury is related to the initial semen quality. The main limitation of this study is the relatively low sample size studied and further studies involving cancer patients are needed to confirm these promising results.

Dr. Laura Kelly Thomson Fertility First, Hurstville Westmead Hospital

University of Sydney Hurstville, Sydney Australia E-mail: lthomson@fertilityfirst.com.au 